\title{
CARACTERIZAÇÃO DO POTENCIAL ANTIMICROBIANO DE Ocotea odorifera (VELLOZO) ROHWER
}

\author{
Júlia Moaraes ROGATTO \\ Matheus Coutinho Costa FERREIRA ${ }^{2}$ \\ Rafaela Mayumi $\mathrm{ONO}^{3}$ \\ Lucas Castro de CARVALHO ${ }^{4}$ \\ Dayane Fernandes SOARES ${ }^{5}$ \\ Daniela Cristina de Macedo VIEIRA ${ }^{6}$ \\ Jorge Kleber CHAVASCO ${ }^{7}$
}

\begin{abstract}
${ }^{1}$ Graduanda em Farmácia, bolsista FAPEMIG, UNIFAL-MG (Universidade Federal de Alfenas) -
ju_rogatto@hotmail.com

${ }^{2}$ Graduando em Farmácia, UNIFAL-MG- matheusccf@yahoo.com.br

${ }^{3}$ Graduanda em Farmácia, UNIFAL-MG- rafa_ono@ hotmail.com

${ }^{4}$ BIC-Jr (FAPEMIG) - lucascdex@ gmail.com

${ }^{5}$ BIC-Jr (FAPEMIG) - dayane_fernandes_soares@ hotmail.com

${ }^{6}$ Professora co-orientadora, Faculdade Pitágoras - danicmvieira@yahoo.com.br

${ }^{7}$ Professor orientador, UNIFAL-MG- jkchavasco@uol.com.br
\end{abstract}

\section{Recebido em: 25/05/2014 - Aprovado em: 30/06/2014 - Disponibilizado em: 30/07/2014}

\begin{abstract}
Resumo: Várias espécies vegetais são utilizadas na medicina popular, no entanto, pouco se conhece sobre seu potencial medicamentoso. Sabendo-se que a resistência dos micro-organismos aos antimicrobianos está crescendo e a descoberta de novos medicamentos é limitada, torna-se importante a busca por novas substâncias originadas de plantas nativas como Ocotea odorifera (sassafrás). Neste trabalho, foi realizada a avaliação da atividade antimicrobiana de extratos de caule, folhas e casca do caule dessa espécie. A avaliação da atividade antimicrobiana e a determinação da concentração inibitória mínima (CIM) foram realizadas por meio da técnica de difusão em ágar e microdiluição em caldo, respectivamente. Os micro-organismos testados foram Bacillus subtilis, Micrococcus luteus, Staphylococcus aureus, Enterococcus faecalis, Escherichia coli, Pseudomonas aeruginosa, Mycobacterium tuberculosis H37, Candida glabrata, Candida parapsilosis, Candida albicans e Saccharomyces cerevisae. Observou-se que houve formação de halos de inibição para as bactérias Gram-positivas com exceção do E. faecalis, que não apresentou halos de inibição assim como as bactérias Gram-negativas. Na determinação da CIM, verificou-se que concentrações entre 0,781 a 6,250 $\mathrm{mg} / \mathrm{mL}$ foram capazes de inibir o crescimento das bactérias Gram-positivas.. No caso das leveduras somente Saccharomyces cerevisae mostrou-se sensível. Não houve formação de halos de inibição para M. tuberculosis. O extrato da casca do caule foi o mais efetivo. Os resultados obtidos permitem concluir que os extratos testados apresentam-se promissores para a obtenção de novas substancias com atividade antimicrobiana, principalmente sobre bactérias Gram-positivas.
\end{abstract}

Palavras-chave: Ocotea odorifera. Sassafrás. Atividade antimicrobiana. CIM. Difusão em ágar.

\section{DESCRIPTION OF OCOTEA ODORIFERA'S (VELLOZO) ROHWER ANTIMICROBIAL POTENTIAL}

Abstract: There are many plants' species being used by folk medicine, though, their drug potential is not very known. Knowing that the antimicrobials microorganisms' resistance is growing, and the discovery of medicines is limited, it becomes important to search new substances from native plants, like Ocotea odorifera (sassáfras). This work tested the antimicrobial activity of $O$. odorifera leaves, stalk and bark. The antimicrobial activity and the minimal inhibitory concentration (MIC) were evaluated by agar diffusion and broth microdilution, respectively. The tested microorganisms 
were Bacillus subtilis, Micrococcus luteus, Staphylococcus aureus, Enterococcus faecalis, Escherichia coli, Pseudomonas aeruginosa, Mycobacterium tuberculosis H37, Candida glabrata, Candida parapsilosis, Candida albicans and Saccharomyces cerevisae. There was halos formation on Gram-positive bacteria, but E. faecalis, which didn't show a inhibition halo like the Gram-negative bacteria. On MIC's test, we could see that concentrations between 0.781 and $6.250 \mathrm{mg} / \mathrm{mL}$ were able to inhibit the Gram-positive bacteria's growth. The only the yeast sensible to any extract was Saccharomyces cerevisae. There wasn't halo formation when M. tuberculosis was tested. The bark's extract was the most effective. We can conclude with these results that the tested extracts are promising on obtaining new antimicrobial substances, especially over Gram-positive bacteria.

Keywords: Ocotea odorifera. Sassafras. Antimicrobial activity. MIC. Agar diffusion.

\section{Introdução}

O Brasil possui, reconhecidamente, uma alta biodiversidade, tanto de ecossistemas como de espécies. Porém, a utilização desses recursos de maneira inadequada tem gerado uma alta degradação dos mesmos e um baixo retorno econômico e social para o país. Neste sentido, pesquisas que buscam o isolamento de princípios ativos, suas identificações, a verificação de seus empregos em atividades da indústria alimentícia, farmacêutica e cosmética, bem como a obtenção de diferentes extratos dos vegetais envolvidos, constituem atividades relevantes (CANSIAN, et al., 2010; SERAFINI et al., 2002).

Dessa forma, sabendo-se que em geral, bactérias têm a habilidade genética de adquirir e transmitir resistência às drogas utilizadas como agentes terapêuticos, que o problema dos microrganismos resistentes está crescendo e a perspectiva para o uso de antibióticos é indefinida, o estudo de plantas com possíveis atividades antimicrobianas é de importância para a exploração sustentável da biodiversidade brasileira e sua aplicação para a produção de conhecimento (CANSIAN, et al., 2010; AMOROSO, 2002; NASCIMENTO et al., 2000).

Ocotea é o maior gênero no Neotrópico com pelo menos 300 espécies, das quais se estima que 120 a 160 espécies ocorram no Brasil (BAITELLO, 2001; VAN DER WERFF, 1996). Algumas espécies são utilizadas na medicina tradicional, incluindo a Ocotea odorifera (Vell.), também chamada de sassafrás, canela funcho e canela cheirosa (LORENZI, 2002).

\section{A $O$. odorifera é reconhecida} fitoquimicamente pela síntese de flavonoides, como o canferol e quercetina, polipropanoides, esteroides e óleos essenciais com sesquiterpenos (COSTA, 2000; LORDELLO et al., 2000). Dentre os componentes desse óleo essencial está o safrol, comercializado no mundo inteiro e utilizado em vários ramos industriais, sendo empregado no preparo de medicamentos com propriedades sudoríficas, antirreumáticas, antissifilíticas, diuréticas e como repelente de mosquitos e fixador em perfumes (LORENZI; 
MATOS, 2002; PINTO JUNIOR et al., 2010).

Sabendo-se do potencial biológico dessa espécie realizou-se a determinação da atividade antimicrobiana de $O$. odorifera.

\section{Materiais e métodos}

As partes frescas de $O$. odorifera (caule, folhas e casca do caule) foram coletadas no município de Alfenas-MG, em março de 2013 no Bairro Vista Grande a

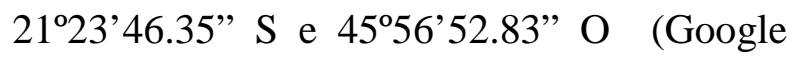
Earth). Foram preparadas exsicatas das mesmas e depositadas no herbário da UNIFAL-MG, identificadas pelo Prof. Dr. Marcelo Polo, recebendo o número 2275. Após fragmentação das partes da planta, a extração foi feita por maceração com agitação diária por sete dias, com etanol a 70\% como liquido extrator. $\mathrm{O}$ extrato foi concentrado em rotaevaporador e liofilizado. A concentração de trabalho foi ajustada em $50 \mathrm{mg} / \mathrm{mL}$ (SILVA et al., 2010).

Os micro-organismos testados foram: Bacillus subtilis ATCC 6633, Micrococcus luteus ATCC 9341, Staphylococcus aureus ATCC 6538, Enterococcus faecalis LMI (UNIFAL), Escherichia coli ATCC 25922, Pseudomonas aeruginosa ATCC 27853, Mycobacterium tuberculosis H37 ATCC 27294, Candida glabrata ATCC 90030, Candida parapsilosis ATCC 22019, Candida albicans ATCC 10231 e Saccharomyces cerevisae ATCC 2601, mantidas no Laboratório de Microbiologia e Imunologia Básicas da UNIFAL-MG..

A atividade antimicrobiana foi avaliada por difusão em ágar segundo metodologia definida nos documento M7A6 (Clinical and Laboratory Standards Institute (CLSI), 2003) para bactérias, M24A2 (2008) para Micobacteria e M44A2 (CLSI, 2009) e M27A3 (2008) para fungos com algumas modificações propostas por Silva et al, (2010). Para as bactérias foi utilizado o Ágar Mueller Hinton e para as leveduras o Ágar Mueller Hinton adicionado de $2 \%$ de glicose, perfurados para obtenção de poços de aproximadamente $4 \mathrm{~mm}$ de diâmetro. Os micro-organismos foram inoculados na superfície do meio de cultura, depois de padronizados em solução salina com turvação correspondente ao tubo 0,5 da escala de Mac Farland para as bactérias e tubo 1,0 para as leveduras. Os extratos foram colocados nos poços, juntamente com os controles positivo (solução de clorexidina a $0,12 \%$ ) e negativo (água destilada). As placas foram incubadas a $37{ }^{\circ} \mathrm{C}$ por 24 horas. Após a incubação, foi feita a leitura dos diâmetros dos halos de inibição do crescimento microbiano com o auxílio de uma régua. 
A atividade sobre $M$. tuberculosis (H37) foi determinada pela técnica de difusão em meio ágar Middlebrook 7H10 adicionado de Middlebrook OADC Enrichment ${ }^{\circledR}$. Os extratos vegetais, num volume de $10 \mu \mathrm{L}$, foram colocados em discos de papel de filtro tipo xarope de $10 \mathrm{~mm}$ de diâmetro e secos a $37^{\circ} \mathrm{C}$. O ágar Middlebrook 7H10 Difco® foi inoculado com uma suspensão de $M$. tuberculosis (H37) com turvação correspondente ao tubo 2,0 da Escala de Mac Farland com auxílio de pérolas de vidro para desagregação das colônias. Foi utilizado como controle positivo solução de Rifampicina $50 \mu \mathrm{g}$ e como controle negativo água destilada (Figura 1). As culturas foram incubadas a $37^{\circ} \mathrm{C}$ por 28 dias.

Todos os ensaios foram realizados em triplicata. Para todos os extratos frente a todos os microrganismos, com exceção do $M$. tuberculosis H37, foi determinada a concentração inibitória mínima (CIM) por microdiluição em caldo conforme metodologia proposta no documento M27A3 (CLSI, 2008) a partir da concentração de 50 $\mathrm{mg} / \mathrm{mL}$ até $0,010 \mathrm{mg} / \mathrm{mL}$.

\section{Resultados e discussão}

Observou-se que houve formação de halos de inibição (Tabela 1) para as bactérias Gram-positivas com exceção do E. faecalis, que não apresentou halos de inibição assim como as bactérias Gram negativas. $\mathrm{O}$ tamanho dos halos para as bactérias Gram positivas variou de 9,67 mm para B. subtilis a 19,33 mm de diâmetro para Micrococcus luteus (Figura 1). Os extratos da casca do caule foram os mais efetivos com os maiores halos de inibição.

Figura 1 - Avaliação da atividade antimicrobiana de extratos de O. odorifera pelo teste de difusão em ágar (resultados dos halos de inibição em mm para M. luteus)

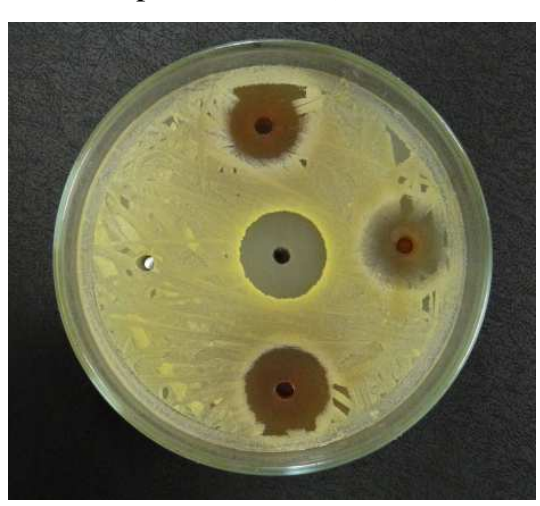

Fonte: os autores.

Na determinação da CIM, verificou-se que para $S$. aureus os valores variaram de 1,17 a $3,13 \mathrm{mg} / \mathrm{mL}$; para B. subtilis a CIM variou de 2,34 a 4,69 $\mathrm{mg} / \mathrm{mL}$ e para Micrococcus luteus os valores variaram de 1,56 a $3,17 \mathrm{mg} / \mathrm{mL}$. No caso das leveduras somente Saccharomyces cerevisae mostrou-se sensível com halos de 12 a 23,33 mm de diâmetro (Tabela 2). Não houve formação de halos de inibição para o $M$. tuberculosis 
(Figura 2). Nas concentrações utilizadas não foram detectados valores de CIM para as bactérias Gram negativas. Os resultados das duas técnicas foram semelhantes, com exceção de Enterococcus faecalis que apresentou valores de CIM de 2,34 a 4,69 $\mathrm{mg} / \mathrm{mL}$ e mostrou-se resistente ao teste de difusão em agar. $\mathrm{O}$ extrato da casca do caule foi o mais efetivo. (Tabela 2)
Figura 2 - Teste de difusão Controle positivo (rifampicina) e negativo (água destilada) do cultivo de M. tuberculosis H37.

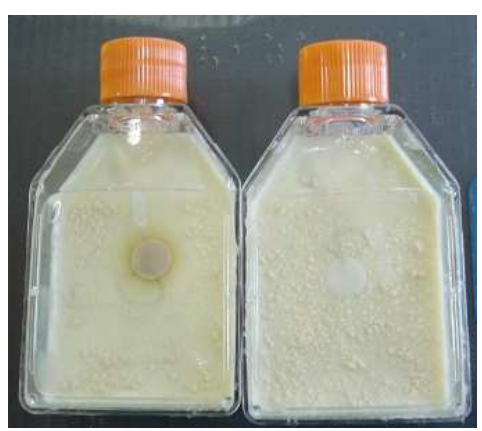

Fonte: os autores.

Tabela 1: Avaliação da atividade antimicrobiana de extratos de O. odorifera pelo teste de difusão em ágar (resultados dos halos de inibição em mm)

\begin{tabular}{lccccc}
\hline \multirow{2}{*}{ Micro-organismos } & \multicolumn{5}{c}{ Extratos e controles } \\
\cline { 2 - 6 } & Caule & Folha & Casca do caule & Água & Clorexidina \\
\cline { 2 - 6 } B.subtilis & 9,67 & 6,33 & 19,33 & 0 & 12,67 \\
M.luteus & 12,67 & 13,33 & 18,67 & 0 & 14,33 \\
S. aureus & 13,33 & 11,33 & 19,0 & 0 & 11,0 \\
E. coli & 0 & 0 & 0 & 0 & 10,0 \\
P.aeruginosa & 0 & 0 & 0 & 0 & 11,33 \\
E. faecalis & 0 & 0 & 0 & 0 & 14,67 \\
C.glabrata & 0 & 0 & 0 & 0 & 15,0 \\
C.parapsilosis & 0 & 0 & 0 & 0 & 13,67 \\
C.albicans & 0 & 0 & 0 & 0 & 12,67 \\
S.cerevisae & 16,33 & 12,0 & 23,33 & 0 & 12,0 \\
\hline Fon & & & & & \\
\hline
\end{tabular}

Fonte: o autores.

Tabela 2 - Determinação da CIM dos extratos de $O$. odorifera pelo teste de diluição em caldo (valores em mg/mL).

\begin{tabular}{lccccc}
\hline Micro-organismos & \multicolumn{5}{c}{ Extratos e controles } \\
\cline { 2 - 6 } & Caule & Folha & Casca do caule & Água & Clorexidina $0,12 \%$ \\
\cline { 2 - 6 } B. subtilis & 2,34 & 4,69 & 3,125 & 0 & 4,69 \\
M.luteus & 3,13 & 3,17 & 1,56 & 0 & 3,17 \\
S. aureus & 1,17 & 3,13 & 1,56 & 0 & 3,13
\end{tabular}




\begin{tabular}{llllll} 
E. coli & ND* & ND & 25,0 & 0 & ND \\
P.aeruginosa & 50,0 & ND & 18,75 & 0 & ND \\
E. faecalis & 2,34 & 3,13 & 4,69 & 0 & 3,13 \\
C.glabrata & ND & ND & ND & 0 & ND \\
C.parapsilosis & ND & ND & ND & 0 & ND \\
C.albicans & ND & ND & ND & 0 & ND \\
S.cerevisae & 2,34 & 3,13 & 4,69 & 0 & 3,13 \\
\hline
\end{tabular}

*ND = Não detectada nas concentrações testadas.

Fonte: os autores.

Estudos similares relatados na literatura apontaram resultados semelhantes ao desse estudo. No trabalho realizado por De Souza e colaboradores (2004), os microorganismos E. coli, B. subtilis, M. luteus, C. albicans e $S$. cerevisae mostraram-se sensíveis ao extrato de $O$. odorifera, enquanto que S. aureus mostrou-se resistente. Já no trabalho de Cansian e colaboradores (2010), os micro-organismos sensíveis ao extrato foram S. aureus, M. luteus, E. faecalis e E. coli, sendo que os dois últimos, em nosso estudo, se mostraram resistentes.

Tais diferenças de resultados podem ser atribuídas a variações nas metodologias empregadas. De acordo com a polaridade de um líquido extrator, moléculas distintas serão extraídas e em diferentes concentrações (Johansen et al., 1996), podendo gerar variados perfis de ação.. O tempo de extração também pode interferir nesse perfil de ação (CUNHA et al., 2004), assim como a região de cultivo e época de coleta do material vegetal (GRACE et AL., 1998). Houve ainda variação nos meios de cultura empregados nos outros trabalhos.

Yamaguchi e colaboradores (2011) extraíram das folhas de $O$. odorifera o elagitanino telimagrandino II, que mostrou-se ativo contra $C$. parapsilosis, indicando que possivelmente as condições do nosso estudo não permitiram a identificação dessa atividade.

\section{Conclusão}

O extrato da casca do caule foi o mais efetivo, entretanto, demais partes da plantas também apresentaram efeitos significativos. Os resultados obtidos permitem concluir que os extratos testados apresentam-se promissores para a obtenção de novas substâncias com atividade antimicrobiana, principalmente sobre bactérias Grampositivas. Estudos posteriores devem ser realizados com enfoque no isolamento de substâncias que desempenhem melhor essa atividade. 


\section{Agradecimentos}

À UNIFAL-MG pelo apoio técnico e a FAPEMIG pelo apoio financeiro.

\section{Referências bibliográficas}

AMOROSO, M. C. M. Uso e diversidade de plantas medicinais em Santo Antônio do Leverger, MT, Brasil. Acta Botanica

Brasilica, v.16, n. 2, p.189-203, 2002.

BAITELLO, J. B. Novas espécies de Lauraceae para a flora brasileira. Acta

Botanica Brasilica, v. 15, n. 3, p. 445-50, 2001.

CANSIAN, R. L.; MOSSI, A. J.; PAROUL, N.; TONIAZZO, G.; ZBORALSKI, F.; PRICHOA, F. C.; KUBIAK, G. B.; LERIN, L. A. Atividade antioxidante e antimicrobiana de extratos de canela-sassafrás (Ocotea odorifera (VELL.) ROWHER).

PERSPECTIVA, Erechim, v. 34, n. 127, p. 123-133, 2010.

\section{CLINICAL AND LABORATORY}

TANDARDS INSTITUTE (CLSI). M24-A2, Susceptibility Testing of Mycobacteria, Nocardiae, and Other Aerobic Actinomycetes. Approved Standard - Second Edition , 2008.

CLINICAL AND LABORATORY
STANDARDS INSTITUTE (CLSI).
Reference method for antifungal disk diffusion susceptibility testing of yeasts. Approved Guideline-Second Edition. M44A2. Wayne, PA, USA: CLSI, 2009.

\section{CLINICAL AND LABORATORY} STANDARDS INSTITUTE (CLSI). Methods for dilution antimicrobial susceptibility tests for bacteria that grow aerobically. Approved standard - Sixth edition. M7-A6. Wayne, PA, USA: CLSI, 2003.

\section{CLINICAL AND LABORATORY} STANDARDS INSTITUTE (CLSI).

Reference Method for Broth Dilution Antifungal Susceptibility Testing of Yeasts. Approved Standard - Third Edition. M27-A3. Wayne, PA, USA: CLSI, 2008.

COSTA, P. R. R. Safrol e eugenol: estudo da reatividade química e uso em síntese de produtos naturais biologicamente ativos e seus derivados. Química Nova, v. 23, n. 3, p. 357-60, 2000.

CUNHA, I. B. S.; SAWAYA, A. C. H. F.; CAETANO, F. M.; SHIMIZU, M. T.; MARCUCCI, M. C.; DREZZA, F. T.; POVIA, G. S.; CARVALHO, P. O. Factors that influence the yield and composition of Brazilian propolis extracts. J. Braz. Chem. Soc., v. 15, n. 6, 2004. 
DE SOUZA, G. C.; HAAS, A. P. S.; VON

POSER, G. L.; SCHAPOVAL, E. E. S.;

ELISABETSKY, E. Ethnopharmacological

studies of antimicrobial remedies in the south of Brazil. Journal of Ethnopharmacology, v. 90, p. 135-143, 2004.

GRACE, S. C.; LOGAN, B. A.; ADAMS, W. W. Seasonal differences in foliar content of chlorogenic acid, a phenylprapanoid antioxidant, in Mahonia repens. Plant, Cell and Environment, v. 21, p. 513-21, 1998.

JOHANSEN, H. N.; GLITS $\varnothing$, V.;

KNUDSEN, K. E. B. Influence of Extraction Solvent and Temperature on the Quantitative Determination of Oligosaccharides from Plant Materials by High-Performance Liquid Chromatography. Journal of Agricultural and Food Chemistry, v. 44, n. 6, p. 1470-4.1996

LORDELLO, A. L. L.; CAVALHEIRO, A. J.; YOSHIDA, M.; GOTTLIEB, O. R. Phenylpropanoids, sterols and sesquiterpene from wood of Ocotea odorifera Lauraceae. Revista Latinoamericana de Química, v. 28, n. 1, p. 35-9, 2000.

\section{LORENZI, H. Árvores brasileiras, manual} de identificação e cultivo de plantas arbóreas nativas do Brasil. Instituto

Plantarum, 2002.

LORENZI, H.; MATOS, F. J. A. Plantas medicinais no Brasil: nativas e exóticas. Instituto Plantarum, 2002.

NASCIMENTO, G.G.F.; LOCATELLI, J.; FREITAS, P.C. Antibacterial activity of plant extracts and phytochemicals on antibiotic resistant bacteria. Brazilian Journal of Microbiology, v. 31, n. 4, p. 247-256, 2000.

PINTO JUNIOR, A. R.; CARVALHO, R. I. N.; NETTO, S. P.; WEBER, S. H.; SOUZA, E.; FURIATTI, R. S. Bioatividade de óleos essenciais de sassafrás e eucalipto em cascudinho. Ciência Rural, v. 40, n. 3, p. 637-43, 2010.

SERAFINI, L. A.; SANTOS, A. C. A.; TOUGUINHA, L. A.; AGOSTINI, G.; DALFOVO, V. Extrações e aplicações de óleos essenciais de plantas aromáticas e medicinais. Caxias do Sul: EDUCS, 2002.

SILVA, R. B.; ALMEIDA, C. R.; CHAVASCO, J. M.; CHAVASCO, J. K. Antimicobacterial Activity Evaluation and MIC Determination of Bixa orellana $\mathrm{L}$ Liophilizated Hydroalcoholic Extracts. 
Revista Brasileira de Farmacognosia, v. 20,

n. 2, p. 171-4, 2010.

VAN DER WERFF, H.; RITCHER, H. G.

Toward and improved classification of

Lauraceae. Annals of the Missouri

Botanical Garden, v. 83, p. 409-18, 1996.

YAMAGUCHI, M. U.; GARCIA, F. P.;

CORTEZ, D. A. G.; UEDA-NAKAMURA,

T.; DIAS FILHO, B. P.; NAKAMURA, C. V.

Antifungal effects of Ellagitannin isolated

from leaves of Ocotea odorifera (Lauraceae).

Antonie van Leeuwenhoek, v. 99, p. 507-14,

2011. 